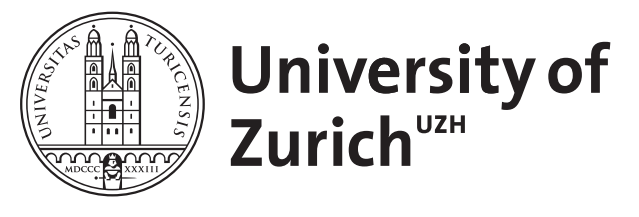

\title{
Enzyme mediated site-specific surface modification
}

\author{
Sala, A ; Ehrbar, M ; Trentin, D ; Schoenmakers, R G ; Vörös, J ; Weber, Franz E
}

\begin{abstract}
Stable tethering of bioactive peptides like RGD to surfaces can be achieved via chemical bonding, biotin streptavidin interaction, or photocross-linking. More challenging is the immobilization of proteins, since methods applied to immobilize peptides are either not specific or versatile enough or might even compromise the protein's bioactivity. To overcome this limitation, we have employed a scheme that by enzymatic (transglutaminase) reaction allows the site-directed and site-specific coupling of growth factors and other molecules to nonfouling poly(L-lysine)-graft-poly(ethylene glycol) (PLL-gPEG) coated surfaces under physiological conditions. By our modular and flexible design principle, we are able to functionalize these surfaces directly with peptides and growth factors or precisely position poly(ethylene glycol) (PEG)-like hydrogels for the presentation of growth factors as exemplified with vascular endothelial growth factor (VEGF).
\end{abstract}

DOI: https://doi.org/10.1021/la1008895

Posted at the Zurich Open Repository and Archive, University of Zurich

ZORA URL: https://doi.org/10.5167/uzh-40388

Journal Article

Accepted Version

Originally published at:

Sala, A; Ehrbar, M; Trentin, D; Schoenmakers, R G; Vörös, J; Weber, Franz E (2010). Enzyme mediated site-specific surface modification. Langmuir, 26(13):11127-11134.

DOI: https://doi.org/10.1021/la1008895 


\title{
Enzyme mediated site-specific surface modification
}

\author{
Ana Sala ${ }^{1,2}$, Martin Ehrbar ${ }^{1,3}$, Diana Trentin ${ }^{4}$, Ronald G. Schoenmakers ${ }^{1}$, Janos Vörös ${ }^{2}$ \\ and Franz E. Weber ${ }^{1}$ \\ ${ }^{1}$ Department of Cranio-Maxillofacial Surgery, Oral Biotechnology \& Bioengineering, \\ University Hospital Zurich and Dental School, University of Zurich. ${ }^{2}$ Institute for Biomedical \\ Engineering, Laboratory of Biosensors and Bioelectronics, ETH Zürich. ${ }^{3}$ Clinic of Obstetrics, \\ University Hospital Zurich. ${ }^{4}$ Laboratory for Surface Science and Technology, Department of \\ Materials, ETH Zurich, Switzerland.
}

\begin{abstract}
Stable tethering of bioactive peptides like RGD to surfaces can be achieved via chemical bonding, biotin streptavidin interaction, or photo-crosslinking. More challenging is the immobilization of proteins, since methods applied to immobilize peptides are either not specific or versatile enough or might even compromise the protein's bioactivity. To overcome this limitation, we have employed a scheme that by enzymatic (transglutaminase) reaction allows the site-directed and site-specific coupling of growth factors and other molecules to non-fouling poly(L-lysine)-graft-poly(ethylene glycol) (PLL-g-PEG) coated surfaces under physiological conditions. By our modular and flexible design principle, we are able to functionalize these surfaces directly with peptides and growth factors or precisely position poly(ethylene glycol) (PEG)-like hydrogels for the presentation of growth factors as exemplified with vascular endothelial growth factor (VEGF).
\end{abstract}




\section{Introduction}

Cells under physiological conditions are embedded within the extracellular matrix (ECM), a viscoelastic highly hydrated network that is mainly composed of glycosaminoglycans and fibrillar proteins. This ECM is not only responsible for structural integrity of tissues but also stores and presents complex spatiotemporal arrays of biochemical and biophysical signals ${ }^{1-4}$ that regulate the dynamic behavior of cells or even whole tissues. Many growth factors are matrix bound and thus, only limited available to cells in quiescent tissues. Furthermore, matrix binding might restrict the diffusion of growth factors from the site of production, protect them from proteolytic degradation, and modulate their activity by coordination of integrin and tyrosine kinase receptor signaling. At this stage we are far from understanding the complex cell-matrix and cell-cell interactions involved in tissue formation, homeostasis, healing, and pathology. In order to study such events and to manipulate cellular behavior, test systems that in a predictable and biologically relevant manner present bioactive entities such as growth factors and cytokines are necessary.

To mimic such complex in vivo environments, recent strategies take advantage of the virtual deconstruction of the ECM components into functional subunits, such as cell adhesion ligands, substrates for proteolytic enzymes, and immobilization of regulatory proteins ${ }^{2,5-6}$. Protein and peptide components are synthesized by either chemical or by recombinant technologies, and incorporated by chemical, physical or biological means in a well-defined fashion either on surfaces ${ }^{7-10}$ or in three-dimensional (3D) matrices ${ }^{2}, 5,11-12$. Since in the naturally occurring situation multiple bioactive signals are presented in a tightly controlled tempero-spatial manner and in variable concentrations, such models should ideally be flexible and versatile enough to specifically, precisely and locally incorporate complex combinations and arrangements of motifs upon a passive background. Most important, mainly regarding incorporation of full proteins, the strategy used to stably immobilize bioligands should be mild enough to not compromise the protein's bioactivity.

While strategies to spatially arrange bioligands in $3 \mathrm{D}$ are only beginning to be explored, modification and patterning strategies on surfaces are much further advanced and have been used to study cell-matrix interactions and to develop biomedical implants with improved healing and performance ${ }^{13}$. For example, surfaces modified with the well-known integrin peptide ligand RGD provided insight into substrate requirements for cell adhesion ${ }^{14-17}$ or cell migration $^{18}$; into nanoscale spatial organization ${ }^{15,19-20}$ and clustering of integrin receptors ${ }^{21-26}$. These studies have become possible due to the advances in micro- and nano-fabrication technologies $8,10,24$ that have allowed for the production of density- and space-controlled 
immobilization of RGD binding peptides on non-fouling substrates like agarose, pluronics, and poly(ethylene glycol) (PEG), to prevent non-specific protein and cell adhesion. The arrangement of RGD peptides has for example been achieved by positioning of gold nanoparticles with immobilized cyclic RGD peptides on a bioinert PEG background. In an elegant system named molecular assembly patterning by lift off (MAPL) ${ }^{27}$, conventional photolithography is employed to create defined nano- and micropatterns of functionalized poly(L-lysine)-graft-poly(ethylene glycol) (PLL-g-PEG) macromers on a non-fouling background. PLL-g-PEG-based copolymers were directly conjugated with cell adhesion ligands, such as $\mathrm{RGD}^{27-29}$, or with biotin which gives the possibility to immobilize different avidin-containing ligands at once ${ }^{30}$.

In contrast to the relatively easy procedures to functionalize passive backgrounds with RGD peptides, the development of platforms that allow for the immobilization and arrangement of proteins, has been hindered by the many obstacles related to protein's specific features. In order to not compromise the stability and activity of the proteins, sufficiently mild strategies that allow for site-specific and stable tethering of ligands which are compatible with the above mention technologies are needed. In recent years, some reports on successful immobilization of growth factors to surfaces by the use of different chemical, physical and biological means have been reported. Ichiose et al. described a direct chemical coupling of epithelial growth factor (EGF) to thiol modified glass-substrates using maleimide reactive groups previously conjugated to primary amine groups of the protein ${ }^{31}$. In another approach EGF was covalently immobilized to the hydroxyl-activated chains of coated poly(methyl methacrylate)-graftpoly(ethylene oxide) (PMMA-g-PEO) on glass slides ${ }^{32}$. Soluble acryol-PEG-transforming growth factor- $\beta 1$ (acryol-PEG-TGF- $\beta 1$ ) has been proven to retain part of its bioactivity when added on glass slides modified with acryolyl-PEG-adhesive peptides ${ }^{33}$. Photo-immobilization strategies have been used to conjugate gradients of EGF and IGF-1 (insulin-like growth factor) modified to contain photoreactive groups onto polystyrene ${ }^{34}$. In an elegant strategy the group of Segura has taken advantage of the heparin-binding activity of vascular endothelial growth factor (VEGF) to "dock" and correctly orient the growth factor on heparin substrates. In a second step they use a photoreactive group to covalently "lock" the growth factor onto the surface ${ }^{35}$. Although chemical reactions have proven to be suitable for the tethering of growth factors to surfaces, the existing immobilization regimen lack versatility and general applicability since they are not compatible with a broad range of growth factors, and often might not result in a site-specific and properly oriented tethering of active proteins. Moreover, it is desirable to integrate such strategies in a micro-patterning context. 
Enzymatic tethering of growth factors to substrates has been reported to be highly specific and due to mild reaction conditions are compatible with protein's functionality ${ }^{36}$. For example transglutaminases, enzymes that rely on $\mathrm{Ca}^{2+}$ and introduce covalent cross-links between glutamine residues (glutamine donor) and lysine residues (glutamine acceptor), have been used to immobilize engineered enzymes or single-chain antibody fragments on amineterminated sepharose or on surfaces previously modified to contain casein in an active state ${ }^{37-}$ ${ }^{38}$. In previous work we have shown that factor XIIIa (FXIIIa), a transglutaminase isoenzyme, can be employed to cross-link star-shaped poly(ethylene glycol) (PEG) polymers that are functionalized with either a glutamine acceptor $(\mathrm{Ac}-\mathrm{F} \underline{K G G})^{39}$ or a glutamine donor $(\mathrm{H}-$ NQEQVSPL) $)^{40}$, here named; Lys substrate or TG substrates respectively, to form synthetic hydrogels ${ }^{41-42}$. The fidelity and specificity of the enzymatic reaction ${ }^{43-45}$, which takes place under physiological conditions is compatible with the protein functionality and could be applied simultaneously to tether growth factors and other ligands to the forming matrix ${ }^{41-42}$. In this study we describe a scheme that relies on the FXIIIa-enzymatic immobilization of biologically active ligands, including peptides and proteins on PLL- $g$-PEG coated surfaces. We show the specificity of the surface modification by Optical Waveguide Light Model Spectroscopy (OWLS) on PLL-g-PEG-based non-fouling surfaces. We demonstrate that this scheme is compatible with the well-established MAPL technology by creation of defined patterns of RGD and VEGF upon a passive PLL- $g$-PEG background. Initial results also indicate that based on consecutive enzymatic reactions, oriented build-up of surfaceimmobilized hydrogel-like structures could be achieved in well-defined positions, giving the possibility to site-specifically deposit relatively large quantities of biologically active substances on a surface. 


\section{Materials and Methods}

All experiments (unless indicated) were performed in HEPES buffer, consisting of $10 \mathrm{mM} 4-$ (2-hydroxyethyl)-piperazine-1-ethane-sulfonic acid and $150 \mathrm{mM} \mathrm{NaCl}$ (Sigma Aldrich, Buchs, $\mathrm{CH}$ ). The $\mathrm{pH}$ of the solution was adjusted to $\mathrm{pH} 7.4$ using $6 \mathrm{M} \mathrm{NaOH}$ (Fluka, Buchs, $\mathrm{CH}$ ). All aqueous solutions were prepared using ultrapure water filtered through MilliQ gradient A 10 filters (Millipore AG, $\mathrm{CH}$ ).

\section{Poly(L-lysine)-graft-poly(ethylene glycol) (PLL-g-PEG) polymers}

Commercially available $\mathrm{PLL}_{20}-g_{3.5}-\mathrm{PEG}_{2}, \quad \mathrm{PLL}_{20}-g_{3.5}-\mathrm{PEG}_{2}-\mathrm{FITC}_{4 \%} \quad$ (SurfaceSolutionS $\mathrm{GmbH}$, Zurich, $\mathrm{CH}$ ) and synthesized $\mathrm{PLL}_{20}-g_{3.5}-\mathrm{PEG}_{2 / 3.4}$-TG were used in this study. The grafting ratio of the polymer $g$, determines the number of lysine monomer units per PEG side chain. This parameter, together with the molecular weight (mol wt) of the poly(L-lysine) backbone and the mol wt of the PEG side chains, were selected on the basis of prior work ${ }^{46}$. This $\mathrm{PLL}_{20}-g_{3.5}-\mathrm{PEG}_{2}$ configuration has been shown to provide excellent performance in terms of resistance to protein adsorption when electrostatically adsorbed on negatively charged oxides.

$\mathrm{PLL}_{20}$ g $_{3.5}-\mathrm{PEG}_{2 / 3.4}$-TG was synthesized starting off from the protocol of VandeVondele ${ }^{47}$ as described in reference ${ }^{13}$. In brief, H-NQEQVSPLERCG-NH $\mathrm{N}_{2}$ peptide (TG substrate) (mol wt 1,358 g/mol, Neosystem, NeoMPS SA, Strasbourg, FRA) and heterofunctionalized PEG, with N-hydroxysuccinimide and vinylsulfone functional termini (NHS-PEG-VS) (mol wt 3,400 $\mathrm{g} /$ mol, polydispersity 1.01, Nektar, Bradford, (UK)), were reacted for $5 \mathrm{~min}$ in a salt buffer solution containing $10 \mathrm{mM}$ HEPES at pH 8.4. Poly(L-lysine) hydrobromide (PLL-HBr) with molecular weight of 20,000 g/mol (polydispersity 1.4, Sigma Aldrich, Buchs, CH) was dissolved in HEPES (10 mM, pH 8.4) and added to the reaction. After one hour Nhydroxysuccinimidyl ester of methoxy terminated poly(ethylene glycol) (mPEG-NHS) (mol wt 2,000 g/mol, polydispersity 1.02, Sigma-Aldrich, Buchs, $\mathrm{CH}$ ) was dissolved in HEPES (10 $\mathrm{mM}, \mathrm{pH} 8.4$ ) and added to the final mixture that was stirred for 24 hours at room temperature. $50 \mu 1$ of $\beta$-mercaptoethanol (Fluka, Buchs, $\mathrm{CH}$ ) was used for quenching and prior to freezedrying, the mixture was dialyzed against deionized water for 48 hours. Synthesized PLL- $g$ PEG-TG resulted in a white powder and was kept frozen at $-20^{\circ} \mathrm{C}$ before use.

Grafting ratio $g$ and fraction of peptide-functionalized PEG-chains were determined by Nuclear Magnetic Resonance Spectroscopy ( ${ }^{1} \mathrm{H}-\mathrm{NMR}$ ) (Supporting information Figures S1, S3). Both the adsorption and the degree of resistance of the polymeric adlayers to serum adsorption were monitored in situ and quantitatively with Optical Waveguide Lightmode Spectroscopy (OWLS) (Supporting information Figure S2). The grafting ratio and the 
adsorbed polymer mass allowed us to determine the surface densities of the immobilized peptide moieties $^{29,48}$.

\section{PEG and peptides}

Star-shaped 8-arm PEG, mol wt 40,000 g/mol, was purchased from Nektar (Huntsville, AL). Divinyl sulfone was purchased from Sigma-Aldrich (Buchs, $\mathrm{CH}$ ). Substrate peptides for FXIIIa; Ac-FKGG-GPQGIWGQ-ERCG-NH 2 (Lys substrate, mol wt 1,718 g/mol) and HNQEQVSPL-ERCG-NH ${ }_{2}$ (TG substrate, mol wt 1,358.5 g/mol), the two adhesion ligands AcFKGG-RGDSP-NH 2 (Lys-RGD, mol wt 1,018.3 g/mol), H-NQEQVSPL-RGDSPG-NH (TG- $^{-}$ RGD, mol wt 1,539.6 g/mol) and the fluorescence ligand Ac-FKGGK(fluorescein)- $\mathrm{NH}_{2}$ (Lys-FITC mol wt $966.2 \mathrm{~g} / \mathrm{mol}$ ) were obtained from NeoMPS (Strasbourg, F). TG-VEGF 121 $(1.36 \mu \mathrm{g} / \mu \mathrm{l}$ dissolved in TBS, $\mathrm{pH}$ 7.4) was provided by P.D. Andreas Zisch (Clinic of Obstetrics, University Hospital Zurich, Zurich, $\mathrm{CH}$ ).

PEG vinylsulfone (PEG-VS) was produced and characterized as described elsewhere ${ }^{49}$. In a second step, PEG-VS were functionalized with Lys and TG substrates via Michel-type addition to give TG-PEG and Lys-PEG precursors. Functionalization and characterization of these precursors was performed as described elsewhere ${ }^{42}$. In brief, peptides were added to PEG-VS in a 1.2 fold molar excess over VS groups in $0.3 \mathrm{M}$ triethanolamine ( $\mathrm{pH} 8.0)$ at 37 ${ }^{\circ} \mathrm{C}$ for $2 \mathrm{~h}$, followed by dialysis (Snake Skin, MWCO 10k, PIERCE, Rockford, IL, USA) against ultrapure water for 4 days at $4{ }^{\circ} \mathrm{C}$.

\section{Activation of FXIII by thrombin}

$100 \mu \mathrm{l}$ of FXIII (200 U/ml, Fibrogammin P1250 CSL Behring) was activated in presence of $2.5 \mathrm{mM} \mathrm{CaCl} 2$ with $10 \mu \mathrm{l}$ of thrombin $(20 \mathrm{U} / \mathrm{ml}$, Sigma-Aldrich, $\mathrm{CH})$ for $30 \mathrm{~min}$ at $37{ }^{\circ} \mathrm{C}$. Small aliquots $(200 \mathrm{U} / \mathrm{ml})$ of activated factor XIIIa were stored at $-80{ }^{\circ} \mathrm{C}$ for further use $^{41-42}$.

\section{Cell culture}

MC3T3-E1 mouse preosteoblastic cells from American Type Culture Collection (ATCC, Manassas, VA, USA) were grown in alpha-minimal essential medium (Gibco, Invitrogen, $\mathrm{CH})$ supplemented with $10 \%(\mathrm{v} / \mathrm{v}) \mathrm{FBS}$ at $37{ }^{\circ} \mathrm{C}$ and $5 \% \mathrm{CO}_{2}$. Cells were trypsinized and passaged every 2 to 3 days. Cultures were never allowed to become confluent.

\section{Optical Waveguide Light Model Spectroscopy (OWLS)}

OWLS is an optical technique that allows for determining the adsorbed mass on a surface based on changes in the refractive index on the vicinity of the surface upon adsorption of molecules from solution. More details about this technique can be found elsewhere ${ }^{50}$. The instrument (OWLS 120) and the waveguides were obtained from MicroVacuum Ltd. 
(Budapest, H). Waveguides were sputter-coated with $6 \mathrm{~nm} \mathrm{TiO}_{2}$ at PSI (Villigen, $\mathrm{CH}$ ). Shortly prior to use, waveguides were cleaned by ultrasonication for $10 \mathrm{~min}$ in 2-propanol and for $10 \mathrm{~min}$ in ultra-pure water, subsequently blown dried with filtered nitrogen and oxygen and plasma cleaned for 2 min (PDC-32 G. Harrick Plasma).

For each experiment, the waveguide was mounted into a flow-through cell (15 $\mu 1$ volume) and HEPES was introduced. After stabilization of the buffer baseline, PLL- $g$-PEG-TG was injected and allowed to adsorb for at least $15 \mathrm{~min}$ before rinsing with HEPES. The protein resistance of the polymer was tested by injection of $3 \%$ bovine serum albumin (BSA) in HEPES. Subsequently, the surface was then exposed for $5 \mathrm{~min}$ to $25 \mathrm{mM} \mathrm{CaCl}_{2}$ in HEPES, rinsed with HEPES and the resistance of the polymer layer tested again with $3 \%$ BSA. Followed stabilization with the new buffer baseline ( $25 \mathrm{mM} \mathrm{CaCl}_{2}$ in HEPES 2), solutions for enzymatic surface modifications were injected and incubated for at least $30 \mathrm{~min}$. To determine the adsorbed mass for each modification we calculated the average mass difference before and after the injection of each solution. The adsorbed mass was calculated according to de Feijter's formula, using a refractive index increment $(\mathrm{dn} / \mathrm{dc})$ of $0.139 \mathrm{~cm}^{3} / \mathrm{g}$ for PEG-based polymers and $0.182 \mathrm{~cm}^{3} / \mathrm{g}$ for proteins.

\section{Molecular Assembly Patterning by Lift off (MAPL)}

MAPL is a technique that allows for the production of micropatterns on a non-fouling surface via combination of conventional photolithography and assembly of functional PEG-graft polycationic copolymers on a negatively charged oxide-coated wafer. More details about this technique can be obtained elsewhere ${ }^{27}$. In brief, a photoresist layer was patterned (squares of $60 \times 60 \mu \mathrm{m})$ on a $\mathrm{Nb}_{2} \mathrm{O}_{5}$-coated MAPL wafer $(\mathrm{ca} .10 \mathrm{~cm})$ by spin coating the surface with a photoresist, UV illumination through a mask and finally development. Afterwards, wafers were diced in 1 x $1 \mathrm{~cm}$ chips. Shortly before use, chips were cleaned by ultrasonication for 10 min in ultra-pure water, dried with filtered nitrogen and oxygen plasma cleaned for 2 seconds. Subsequently either PLL- $g$-PEG-TG $(0.5 \mathrm{mg} / \mathrm{ml})$ or PLL- $g$-PEG-FITC $(0.5 \mathrm{mg} / \mathrm{ml})$ were immobilized on the unprotected areas of the substrate by spontaneous assembly from aqueous solution, followed by lift off of the photoresist in 1-methyl-2-pyrrollidone (NMP). In order to render the surface resistant to non-specific protein adsorption, bare metal oxide areas were backfilled with PLL- $g$-PEG.

PLL-g-PEG, PLL-g-PEG-FITC and PLL-g-PEG-TG stocks (lyophilized white powder, kept frozen at $-20^{\circ} \mathrm{C}$ ) were equilibrated at room temperature for $5 \mathrm{~min}$ prior to use and dissolved in HEPES 2 buffer to reach a final concentration of $0.5 \mathrm{mg} / \mathrm{ml}$. Solutions were stored at -20 ${ }^{\circ} \mathrm{C}$ and warmed up to room temperature before use. 


\section{Factor XIIIa-catalyzed coupling of bioligands onto the surface}

The TG-pendant domains patterned on the surface of the MAPL chips (squares of $60 \times 60$ $\mu \mathrm{m})$ were used to couple ligands containing a Lys substrate domain via factor XIII (FXIIIa) enzyme-catalyzed reaction to the surface. The MAPL wafers were incubated with either 100 $\mu 1$ of i) Lys-FITC $(50 \mu \mathrm{M})$, ii) Lys-RGD $(50 \mu \mathrm{M})$ or iii) 8-arm Lys-PEG $(200 \mu \mathrm{M})$ for $30 \mathrm{~min}$ at room temperature in presence of calcium $\left(\mathrm{CaCl}_{2}, 25 \mathrm{mM}\right)$ and FXIIIa $(5 \mathrm{U} / \mathrm{ml})$. Then the samples were rinsed 3 times for $5 \mathrm{~min}$ in HEPES and subsequently ultrasonicated for $2 \mathrm{~min}$. i) Lys-FITC patterns were evaluated with the help of an epifluorescence microscope (Zeiss Microscope, Hamamatsu EM-CCD digital camera). As a negative control for the Lys-FITC pattern, a competitive assay was performed with soluble TG-RGD $(200 \mu \mathrm{M})$ that competed with the TG residues on the surface. ii) Investigation of cell adhesion on the Lys-RGD patterns was performed in medium supplemented with $0.1 \%$ fetal bovine serum and antibiotics (100 U/ml penicillin $\mathrm{G}$ and $100 \mathrm{mg} / \mathrm{ml}$ streptomycin). MC3T3-E1 preosteoblastic cells were cultured at a density of 10,000 cells $/ \mathrm{cm}^{2}$ for 4 hours and subsequently fixed and stained for f-actin as described below. As a negative control, a pattern of PLL- $g$-PEG-FITC on a PLL- $g$-PEG background was incubated with $100 \mu$ of Lys-RGD $(50 \mu \mathrm{M})$ in presence of FXIIIa $(5 \mathrm{U} / \mathrm{ml})$ and calcium $\left(\mathrm{CaCl}_{2}, 25 \mathrm{mM}\right)$ for $30 \mathrm{~min}$ at room temperature. iii) The 8-arm Lys-PEG tethered to the surface presenting still free, non-bound Lys domains was used in a second reaction step for FXIIIa-catalyzed immobilization of TG-VEGF T21 $_{1}(5.4 \mu \mathrm{g}$ in $100 \mu \mathrm{l}$ in HEPES). Binding of the growth factor to the surface was proved with VEGF specific first antibody (rabbit polyclonal VEGF (A-20) sc-152, Santa Cruz Biotechnology) followed by alexa fluor 555 goat anti-rabbit secondary antibody (A-21428, Invitrogen). As a negative control 8-arm Lys-PEG was not immobilized on the surface.

\section{Fixation and Staining Protocol}

MC3T3-E1 preosteoblastic cells were fixed and permeabilized in 4\% paraformaldehyde containing $0.2 \%$ Triton $\mathrm{X}-100$ in phosphate buffered saline (PBS) for $20 \mathrm{~min}$ at room temperature. Samples were washed for $5 \mathrm{~min}$ with $0.1 \mathrm{M}$ glycine in PBS followed by a second washing step in PBS. For f-actin staining, samples were incubated (protected from light) with $0.4 \mathrm{U} / \mathrm{ml}$ rhodamine-labeled phalloidin (Molecular Probes, Eugene, OR, USA) in PBS 1\% BSA O.N. at $4{ }^{\circ} \mathrm{C}$. Afterwards samples were washed 3 times for $5 \mathrm{~min}$ in PBS at room temperature and stored till evaluation. 


\section{Results and Discussion}

FXIIIa, involved in the fibrin clot formation, is an activated transglutaminase that catalyzes an acyl-transfer reaction between the R-carboxamide group of protein-bound glutaminyl (Gln, or $\mathrm{Q}$ in the single letter nomenclature) residues and the $\varepsilon$-amino group of lysyl (Lys, or K) residues, resulting in the formation of $\varepsilon$-( $\alpha$-glutamyl) lysine isopeptide side-chain bridges ${ }^{44-45}$. In the initial step, FXIIIa interacts in a specific manner with the glutamine substrate which results in the formation of a thioester bond between the enzyme and the substrate and the release of ammonia. Afterwards, this enzyme-glutamine complex binds to the primary amine of the lysine containing substrate leading to an isopeptide bridge between the glutamine and the lysine, with the subsequent release of the enzyme ${ }^{43-44}$. In previous work we have shown that FXIIIa can be employed to crosslink star-shaped PEG polymers that are functionalized with either Lys or TG substrates to form synthetic hydrogels. Moreover, we have shown that the FXIIIa-catalyzed coupling scheme allows for the covalent incorporation of growth factors during the formation of these PEG hydrogels, without compromising the protein's bioactivity ${ }^{41-42}$. Here, we aim to use this enzymatic reaction to site-specifically immobilize growth factors or other bioactive molecules on a surface.

\section{Design of the surface modification scheme}

To probe the specificity of the immobilization scheme we developed an approach that takes advantage of the above mentioned patterning technique; MAPL. In a first step, as depicted in Figure 1a, this MAPL technique ${ }^{27}$ is used to generate a pattern of PLL- $g$-PEG-TG $(60 \times 60 \mu \mathrm{m}$ squares) in a non-fouling PLL-g-PEG background on a $\mathrm{Nb}_{2} \mathrm{O}_{5}$-coated surface. Next (Figure 1b), the TG-pendant domains of the PLL- $g$-PEG-TG copolymer are employed for tethering of ligands designed to contain Lys-substrates, here 8-arm Lys-PEG, by FXIIIa to the surface. In a subsequent step, ligands that contain the counter reactive substrate for FXIIIa, TG substrate, are immobilized by the same reaction scheme to the immobilized non-reacted Lys substrates. Thus, by controlling the position of the TG residues on the surface and translating this pattern via the FXIIIa-enzymatic reaction into a bioactive pattern, it is possible to have control over the arrangement of the bioligands. 
a



b

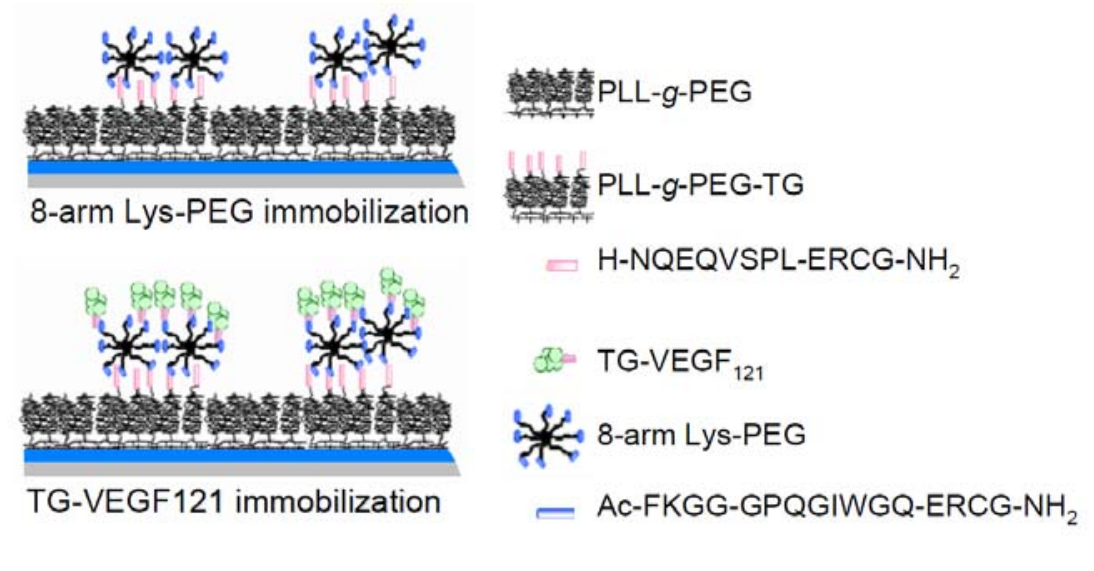

Figure 1. Illustration of the scheme of factor XIIIa-catalyzed coupling of bioligands on a surface. (a) PLL-gPEG-TG is patterned on a non-fouling PLL-g-PEG background by Molecular Assembly Patterning by Lift-off (MAPL). (b) The TG pendant domains on the surface are used for enzymatic immobilization of bioligands that have been previously engineered to contain a factor XIIIa substrate sequence. Here, 8-arm Lys-PEG star-shaped macromers are bound to the surface. In a second step, vascular endothelial growth factor engineered to contain the TG sequence $\left(\mathrm{TG}_{-} \mathrm{VEGF}_{121}\right)$ is linked to the immobilized non-reacted Lys Substrates.

\section{Synthesis and characterization of PLL-g-PEG-TG}

In order to position molecules on a surface, patterning strategies such as photolithographic, microfluidic, electrochemical techniques can be used. The PLL- $g$-PEG technology besides providing non-fouling properties to negative charged surfaces such as metals ${ }^{29,46,48}$, has been used together with MAPL to spatially arrange biologically active molecules like RGD on these surfaces and thus enable selective cell adhesion ${ }^{27}$.

To allow the enzyme FXIIIa to locally and specifically interact with the protein-resistant surface coating, we functionalized the PLL-g-PEG copolymer with a pending TG substrate sequence (PLL- $g$-PEG-TG). PLL- $g$-PEG-TG was synthesized and characterized by ${ }^{1} \mathrm{H}-\mathrm{NMR}$ and OWLS (data included in Supporting information Figures S1, S2, S3). ${ }^{1} \mathrm{H}-\mathrm{NMR}$ rendered a grafting ratio of 4.4 and a content of peptide-functionalized PEG-chains versus total amount of PEG-chains of $25 \%$. In situ measurement on a titanium-coated waveguide by OWLS rendered an adsorbed polymer mass of $c a .132 \pm 5 \mathrm{ng} / \mathrm{cm}^{2}$ and a high degree of resistance of these polymeric adlayers to serum adsorption, shown by only $c a .1 .8 \pm 1.2 \mathrm{ng} / \mathrm{cm}^{2}$ protein adsorption. Both values were consistent with earlier publications ${ }^{29,48}$. Based on the grafting ratio and the adsorbed polymer mass of our polymer, we calculated the surface density of the 
immobilized TG-peptide moieties and the distance between them on a titanium-coated surface, and obtained $9.1 \mathrm{pmol} / \mathrm{cm}^{2}$ and $4.6 \mathrm{~nm}$ respectively, similar to values of peptide moieties reported by other groups ${ }^{27-29}$.

\section{Patterning of FITC- labelled Lys substrates}

Since the coating of titanium-coated surfaces with PLL-g-PEG-TG proved to be resistant to protein adsorption (Supporting information Figure S2), we next created patterns of functionalized PLL-g-PEG-TG copolymers in a non-fouling PLL- $g$-PEG background by MAPL. We wanted to test whether the TG domains on the surface of PLL-g-PEG were accessible for tethering of biomolecules by FXIIIa. Therefore, we designed a short peptide sequence consisting on the TG-counter reactive substrate for FXIIIa followed by a short linker and fluorescein; Lys-FITC, which allowed us to directly follow the coupling reaction to the free pending TG peptide on the surface. After the reaction of Lys-FITC $(50 \mu \mathrm{M})$ in presence of FXIIIa and calcium ions, the surfaces were washed thoroughly and analyzed by epifluorescence microscopy. In initial experiments the corresponding pattern of $60 \times 60 \mu \mathrm{m}$ green squares (false colored), separated by $100 \mu \mathrm{m}$, could be visualized at a wavelength of $470 \mathrm{~nm}$ on a dark background (Figure 2a). As negative control we performed a competitive assay using a large excess of soluble TG over bond TG substrates. The addition of TG-RGD $(200 \mu \mathrm{M})$ in the Lys-FITC $(50 \mu \mathrm{M})$ mixture resulted in a surface with an undetectable fluorescence pattern (Figure 2b). These results imply that the TG domains on the surface were still functional and accessible for FXIIIa and that binding of the Lys-FITC molecules to the surface was indeed mediated by FXIIIa activity.

\section{Enzymatic tethering of the integrin binding ligand RGD}

Next, we wanted to test whether this modification scheme could be used to tether biologically relevant molecules. Previous findings indicated that functionalized PLL-g-PEG-RGD on metal implant surfaces sustained the adhesion of cells and could be used for tissue engineering applications such as the development of biomedical implants with improved healing and performance in vivo ${ }^{13,29}$. To prove the specific coupling of Lys-RGD to PLL- $g$ PEG-TG coated surface via FXIIIa reaction, $\mathrm{Nb}_{2} \mathrm{O}_{5}$-coated surfaces were patterned with either PLL- $g$-PEG-TG or PLL- $g$-PEG-FITC on a cell- and protein-resistant background. After incubation with the Lys-RGD containing reaction mix, the non-bound ligands were carefully removed by rinsing. The presence and availability of the RGD peptide was assessed by allowing adhesion of MC3T3-E1 preosteoblastic cells for 4 hours under low serum $(0.1 \%)$ cell culture conditions. As expected, cells exclusively attached to the areas were PLL- $g$-PEGTG was immobilized (Figure 2c) and neither to the immobilized PLL- $g$-PEG-FITC (Figure 
2d) nor to the PLL-g-PEG background, as shown by epifluorescence microscope imaging. These results prove that the squares covered by PLL- $g$-PEG-TG translate into bio-adhesive patterns (RGD), and that the Lys-RGD ligands tethered to the surface are available to the cells.
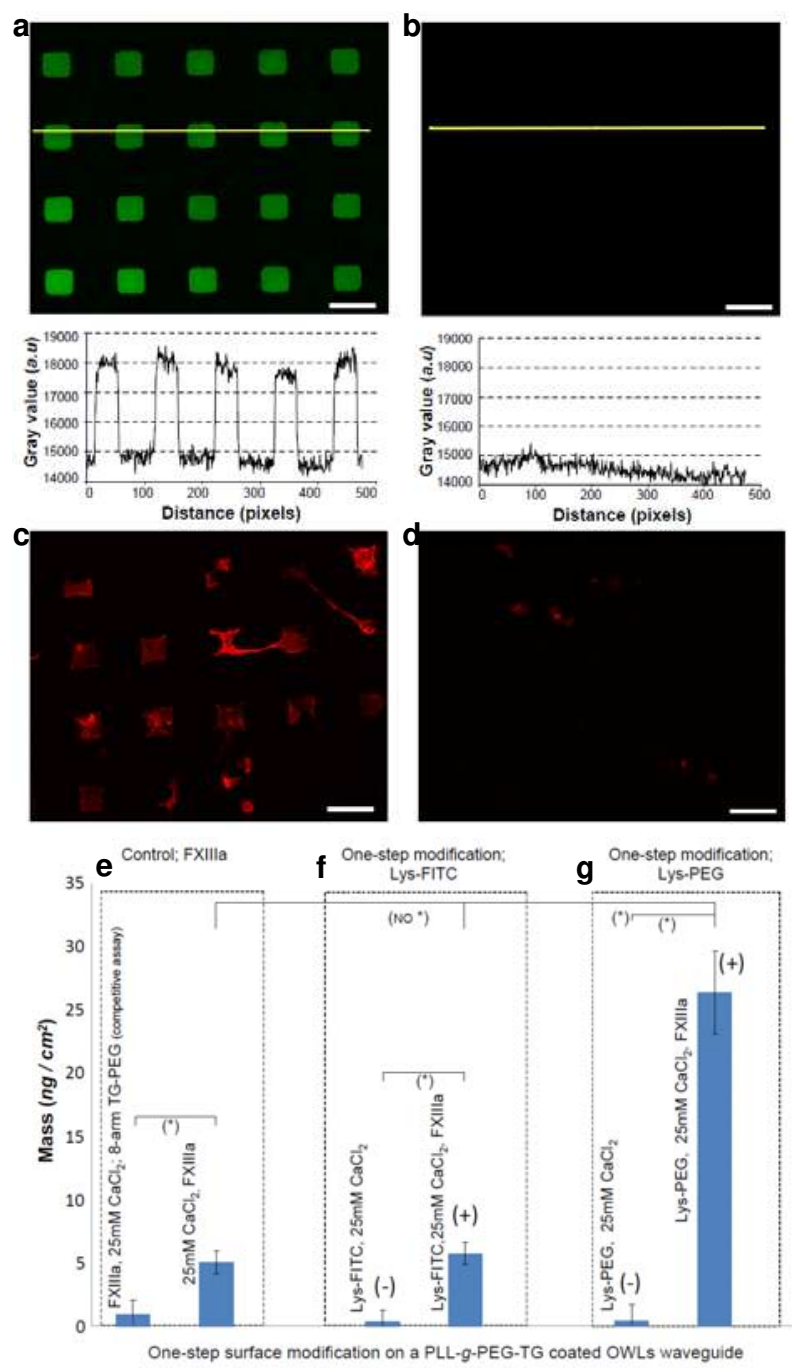

Figure 2. Enzymatic immobilization of Lys modified substrates on a surface by MAPL and OWLS. (a) 60x60 $\mu \mathrm{m}$ pattern of FITC-labeled Lys substrate; (b) same in the presence of soluble TG-RGD in the incubation mixture (competitive assay); (c) MC3T3 cells on the RGD-functionalized pattern, (d) cells on a pattern without the RGD functional group. Fluorescence intensity (in gray scale, original colored images) was measured with "ImageJ's Plot Profile". The scale bar corresponds to $100 \mu \mathrm{m}$. (e-g) In situ surface immobilization of different ligands on PLL-g-PEG-TG coated waveguides by OWLS (three independent experiments, (-) control, (+) positive sample, $\left({ }^{*}\right)$ significantly different, $\left(\mathrm{NO}^{*}\right)$ no significantly different (statistical analysis performed "dunn test"), $\mathrm{n}=3$ ).

\section{Theoretical RGD ligand density}

In this study we did not evaluate the influence of the RGD ligand density on cell spreading or focal adhesion formation. However, we calculated the maximum theoretical Lys-RGD surface 
peptide density and the distance between the moieties on both surfaces ${ }^{29}$, based on the polymer structure and the adsorbed PLL- $g$-PEG-TG mass on the surface $\left(132 \mathrm{ng} / \mathrm{cm}^{2}\right.$ on $\mathrm{TiO}_{2}$-coated surfaces (measured by OWLS), $170 \mathrm{ng} / \mathrm{cm}^{2}$ on $\mathrm{Nb}_{2} \mathrm{O}_{5}$-coated surfaces (from bibliography $\left.{ }^{27}\right)$. We obtained a density of $9.1 \mathrm{pmol} / \mathrm{cm}^{2}\left(9.2 \mathrm{ng} / \mathrm{cm}^{2}\right)$ and a distance of $4.6 \mathrm{~nm}$ for $\mathrm{TiO}_{2}$-coated surfaces and a density of $11.7 \mathrm{pmol} / \mathrm{cm}^{2}\left(11.9 \mathrm{ng} / \mathrm{cm}^{2}\right)$ and a distance of $4 \mathrm{~nm}$ for $\mathrm{Nb}_{2} \mathrm{O}_{5}$-coated surface (Supporting information Figure S3). Such values are in the range of reported values on PLL- $g$-PEG-RGD copolymer $\left(3.36 \mathrm{pmol} / \mathrm{cm}^{2}\right.$ and $\left.8 \mathrm{~nm}\right)$ that have been proven to activate focal adhesion sites on titanium surfaces ${ }^{29}$. Moreover, integrin $\alpha 5 \beta 3$ mediated fibroblast spreading has been reported to occur at a mean RGD peptide spacing of $440 \mathrm{~nm}$ (corresponding to $0.001 \mathrm{pmol} / \mathrm{cm}^{2}$ ), while focal contact formation and extensive $\mathrm{f}$ actin stress fibers has been reported to require a mean spacing of $\leq 140 \mathrm{~nm}$ (corresponding to $\left.0.01 \mathrm{pmol} / \mathrm{cm}^{2}\right)^{14}$. That such $\mathrm{Nb}_{2} \mathrm{O}_{5}$-coated wafers remain stable over more than ten days, and thus can be used to culture cells, has been shown in defined patterns of PLL-g-PEG-RGD and PLL-g-PEG ${ }^{51}$.

\section{In situ surface modification by $O W L S$}

Besides testing the specificity of FXIIIa surface modification by MAPL, we also wanted to determine the amount of ligands that can be coupled to our surfaces by FXIIIa enzymatic reaction. Thus, we monitored the adsorption of ligands on PLL-g-PEG-TG-coated waveguides $\left(\mathrm{TiO}_{2}\right.$-coated) in situ by OWLS (Supporting information Figure S4 for a representative OWLS experiment; two immobilization steps). Prior to enzymatic modification of PLL- $g$-PEG-TG-coated surfaces, we tested the resistance of the coated-surfaces to BSA $(3 \%)$ and to the effect of calcium ions $(25 \mathrm{mM})$ (data not shown). Experiments shown in Figure $2 \mathrm{e}-\mathrm{g}$ correspond to three independent experiments and include the adsorption values $\left(\mathrm{ng} / \mathrm{cm}^{2}\right)$ of each molecule $(\mathrm{n}=3)$ with the corresponding standard deviation (SD). As mentioned before, FXIIIa first interacts in a specific manner with the TG domain, resulting in the formation of an enzyme-glutamine complex and the subsequent interaction of the complex with a Lys domain. At this stage the stability of this complex in absence of the Lys substrate is unclear. Based on previous work ${ }^{44}$ and our own reported observations ${ }^{42}$ the modification on the TG substrate due to the hydrolysis of one glutamine residues into a glutamic acid residue is occurring. This hydrolysis might not only affect the affinity of FXIIIa for the TG sequence but more importantly might induce the complete inactivation of the substrate. In a first set of experiments we tested the interaction of FXIIIa with the PLL-g-PEG-TG-coated surface. The increase in the mass adsorbed on the surface in response to FXIIIa application $(5.1 \pm 0.9$ $\mathrm{ng} / \mathrm{cm}^{2}$ ) indicates the interaction between the enzyme and the TG substrate in absence of Lys 
substrate. The specificity of this interaction could be demonstrated by the absence of adsorbed mass $\left(0.9 \pm 1.1 \mathrm{ng} / \mathrm{cm}^{2}\right)$ upon addition of 8 -arm TG-PEG $(400 \mu \mathrm{M})$ to the reaction mixture (Figure 2e). In the next set of experiments, the binding of Lys-FITC was assessed in conditions that either did not contain or contained FXIIIa (Figure 2f). As expected, an increase in mass $\left(5.7 \pm 0.9 \mathrm{ng} / \mathrm{cm}^{2}\right)$ consistent with the theoretical calculated mass $(8.8$ $\mathrm{ng} / \mathrm{cm}^{2}$. Supporting information Figure S3) was observed only when FXIIIa was present. However since Lys-FITC is a small molecule $(966.2 \mathrm{~g} / \mathrm{mol})$, this increase was not significantly different from the one obtained for FXIIIa. Therefore, in the next set of experiments (Figure $2 \mathrm{~g}$ ) we modified the surface with the $c a .55$ fold larger star-shaped 8-arm Lys-PEG $(53,744 \mathrm{~g} / \mathrm{mol})$ under the same reaction conditions. A significant increase in mass $\left(26.4 \pm 3.3 \mathrm{ng} / \mathrm{cm}^{2}\right)$ was detected only in presence of FXIIIa. This value corresponds to lower molar coverage than the one we would expect when considering the theoretically available number of TG domains on the surface. The reason for this low mass increase could be explained considering the size of the molecule. Our theoretical calculations assumed the best hypothetical conditions which are; excess of Lys substrate (ensured it in our experimental design), functionality of glutamine substrate on the surface (previously proved by MAPL), complete accessibility of the TG substrates, and $100 \%$ efficiency in the enzymatic reaction. Based on the polymer configuration and on previous work by other groups ${ }^{27}$, the probability that a fraction of TG ligands on the surface, is embedded within the dynamic PEG-brush during the $30 \mathrm{~min}$ incubation period is very low. The coupling efficiency of the enzyme based on previous work should be $c a .80-85 \%{ }^{41-42}$. This later reasoning is supported by the in situ increase in mass measured for Lys-FITC, a small molecule which diameter we assumed to be $<4.6 \mathrm{~nm}$ (theoretical distance between TG moieties $c a .4 .6 \mathrm{~nm}$ ). In the case of 8-arm LysPEG (ca. 20-30 $\mathrm{nm}$ in diameter), we believe that each molecule tethered to the surface blocks the access of $c a$. 10 TG moieties due to steric hindrance. Both this steric hindrance and the enzymatic efficiency, could explain the mass adsorption quantified by OWLS in the case of 8arm Lys-PEG (Supporting information Figure S3).

\section{Surface immobilization of vascular endothelial growth factor by MAPL and OWLS}

Once we proved that the TG domains immobilized on the surface can be used for the sitespecific enzymatic binding of ligands, we wanted to proceed by the building up of a second layer of macromers in a sequential fashion as depicted in Figure 1b. The stepwise coupling of layers would be useful for the flexible and modular design of surface properties, for example for immobilization of growth factors in well defined positions. To test the specific incorporation of our prototypic growth factor TG-VEGF 121 (Figure $1 \mathrm{~b}$ scheme) we prepared 
two different MAPL substrates. In the first coupling step, PLL- $g$-PEG-TG patterns $(60 \times 60$ $\mu \mathrm{m})$ were reacted in presence or in absence of star-shaped 8-arm Lys-PEG which should result in surfaces that did or did not contain free surface-exposed Lys substrates. If these substrates are accessible for FXIIIa they should in a next reaction step be available for the linkage of a further layer. Therefore, substrates decorated with free Lys domains when reacted in presence of TG-VEGF $121(5.4 \mu \mathrm{g})$ and FXIIIa should present the growth factor in an active and properly oriented fashion. The surface immobilization and distribution of TG-VEGF 121 was proved with a polyclonal rabbit anti-VEGF antibody followed by a goat anti-rabbit IgG labeled with alexa 555. As expected 60x60 $\mu \mathrm{m}$ squares (with some inhomogeneities) contrasted clearly with a dark background in the samples that contained the Lys-PEG layer, while an uniform background was observed for the negative sample and the non-fouling regions from the positive sample (Figures 3a-b). These results indicate a site-specific tethering of TG-VEGF 121 on the regions where the corresponding substrate for FXIIIa (Lys-PEG) was previously immobilized.

The build-up of the surface layers was then confirmed by OWLS. First the tethering of 8-arm Lys-PEG was followed $\left(26.4 \pm 3.3 \mathrm{ng} / \mathrm{cm}^{2}\right)$. Afterwards the surface was exposed to FXIIIa and calcium, as expected; no significant mass increase was detected $\left(0.06 \pm 1.1 \mathrm{ng} / \mathrm{cm}^{2}\right)$. On the contrary, a significant mass increase of $10.1 \pm 3 \mathrm{ng} / \mathrm{cm}^{2}$ was measured when TG-VEGF 121 was added to the reaction mixture (Figure 3c).

To discuss TG-VEGF 121 immobilization values we first consider how Lys-PEG is presented on the surface. When considering that the 8-arm Lys-PEG molecule will present a cubical geometry with each arm directed towards one of the 8 vertex of the cube, the edge length would be in the range of 11.6 to $17.3 \mathrm{~nm}$ (arm size of Lys-Peg ca. $10-15 \mathrm{~nm}$ ). Based on the surface area occupied by one Lys-PEG molecule $\left(11.6^{2}\right.$ to $\left.17.3^{2}\right)$ and assuming whole coverage of the surface, the total amount of Lys-PEG molecules that fit per $\mathrm{cm}^{2}$ will range from 3.3E11 (molecule diameter; $20 \mathrm{~nm}$ ) to 7.4E11 (molecule diameter; $30 \mathrm{~nm}$ ); which is comparable to the experimental number of Lys-PEG molecules $\left(26.4 \pm 3.3 \mathrm{ng} / \mathrm{cm}^{2}, 2.9 \mathrm{E} 11\right.$ molecules). As already suggested we believe that only one out of 10 TG moieties is occupied by an 8-arm Lys-PEG molecule (total TG density: $9.1 \mathrm{pmol} / \mathrm{cm}^{2}\left(9.2 \mathrm{ng} / \mathrm{cm}^{2}\right) ; 1 / 10$ of total density and $80 \%$ efficiency on the surface represents 4.4E11 Lys-PEG molecules). Therefore, we believe that the surface is saturated with a monolayer of Lys-PEG molecules. At this stage we don't know how many Lys motifs at the terminus of each PEG will be accessible by flexibility and molecular dynamics for further modifications by the FXIIIa enzymatic reaction. Calculations for TG-VEGF 121 immobilization (as well as for TG-PEG) indicate that 
$c a$. one dimeric growth factor interacts with one 8-arm Lys-PEG molecule $\left(15.7 \pm 2 \mathrm{ng} / \mathrm{cm}^{2}\right.$ of TG-VEGF $_{121}$ expected when $1 \mathrm{~mol}$ of 8-arm Lys-PEG interacts with $1 \mathrm{~mol}$ of TG-VEGF 121 ; and $62.9 \pm 7.9 \mathrm{ng} / \mathrm{cm}^{2}$ when $1 \mathrm{~mol}$ of 8 -arm Lys-PEG interacts with $4 \mathrm{~mol}$ of TG-VEGF 121 (Supporting information Figure S3). As we have used excess of TG-VEGF 121 (5.4 $\mu \mathrm{g}$ ) for the immobilization, we assume that the amount of immobilized growth factor cannot be further increased. When considering the diameter of TG-VEGF 121 ( $\mathrm{mol}$ wt 32,000 g/mol), based on reported VEGF crystal structures ${ }^{52-53}$, to be in the range of $5 \mathrm{~nm}$ the limited growth factor coupling is most likely not due to sterical hindrance. There is the possibility that one homodimeric TG-VEGF 121 molecule interacts with more than two Lys moieties belonging to either the same or to a different 8-arm Lys-PEG molecule. However, at this stage we cannot provide enough evidence to discuss further scenarios that could explain the observed immobilization ratio.
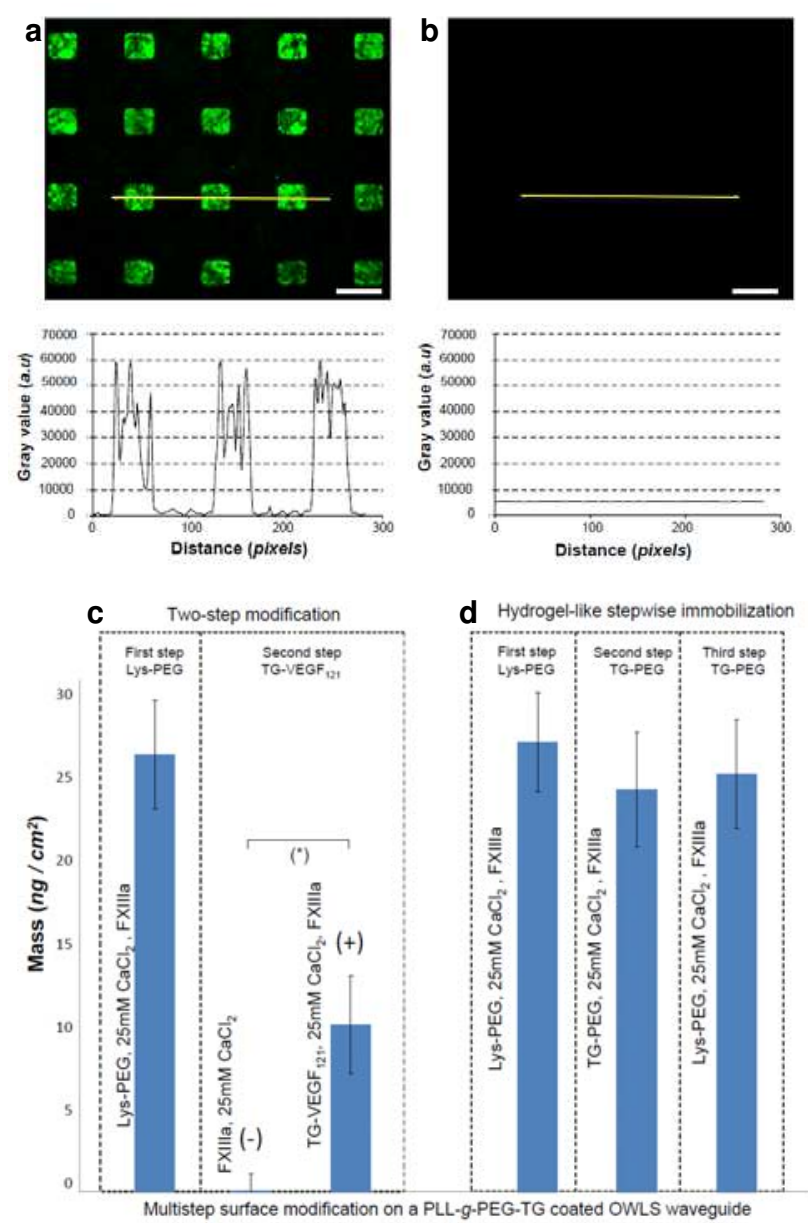

Figure 3. Enzymatic immobilization of vascular endothelial growth factor $\left(\mathrm{TG}-\mathrm{VEGF}_{121}\right)$ by MAPL and OWLS. Presence of TG-VEGF 121 on MAPL samples was detected with a polyclonal rabbit anti-VEGF antibody and a goat anti-rabbit IgG labelled with alexa 555. 60x60 $\mu \mathrm{m}$ squared pattern (green, false colored) was detected in the positive sample (a) but not in the control sample (b) in absence of Lys-PEG. Fluorescence intensity (in gray scale, original colored images) was measured with "ImageJ's Plot Profile" function. The scale bar corresponds to 
$100 \mu \mathrm{m}$. (c-d) In situ stepwise surface immobilization of different ligands on PLL-g-PEG-TG coated waveguides by OWLS (two independent experiments $(-)$ control, $(+)$ positive sample, $\left(^{*}\right)$ significantly different (statistical analysis performed "dunn test"), $n=3$ ).

\section{Stepwise formation of surface immobilized hydrogel-like structures}

Since free and functional substrate domains were observed after individual reaction steps, we followed the build-up of a "layer-by-layer" PEG-like hydrogel. Such a system could be used to form well-organized surface immobilized growth factor depots. The stepwise FXIIIacatalyzed immobilization of Lys-PEG $\left(27.1 \pm 3 \mathrm{ng} / \mathrm{cm}^{2}\right)$ followed by TG-PEG $(24.2 \pm 3.5$ $\left.\mathrm{ng} / \mathrm{cm}^{2}\right)$ and subsequently by Lys-PEG $\left(25.1 \pm 3.3 \mathrm{ng} / \mathrm{cm}^{2}\right)$ was monitored by OWLS. We observed a constant increment of mass for all the steps (Figure 3d). Also for this scenario, we believe to reach the maximum amount that can be immobilized. As previously discussed, after the first immobilization with Lys-PEG we consider our surface to be saturated with a monolayer of Lys-PEG molecules. For the two subsequent immobilization steps (TG-PEG and Lys-PEG) our calculations indicate an interaction ratio of 1 to 1 , which suggests that a monolayer of PEG is being immobilized (Figure 4a) with each step.

In Figure $4 \mathrm{~b}$ we contemplate a possible scenario with multifunctional patterned 3D hydrogellike structures. We exemplify the immobilization of a first layer of Lys-PEG followed by a layer of TG-PEG and a third layer of Lys-PEG. The two first PEG molecules have been previously modified to contain one ligand (same or different) per molecule. The presented platform, as it is flexible in design, could include PEG molecules variable in number and size of arms. Furthermore, PEG molecules could be functionalized with ligands prior to or directly upon immobilization to the surface. In combination with other patterning techniques this layer-by-layer approach could even be used on hydrogel substrates. Together, we think that this stepwise build-up of hydrogel-like structures extends the possibilities of surface confined growth factor patterns to more complex 3D arrangement of biomimetic surface immobilized hydrogel-like substrates. 
a

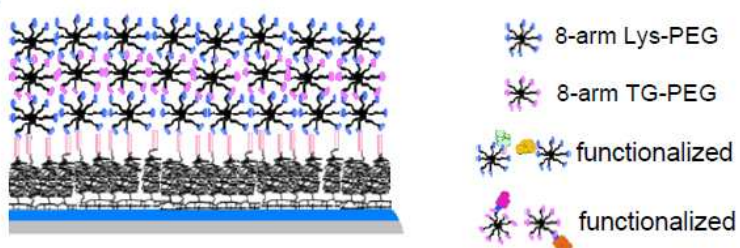

b

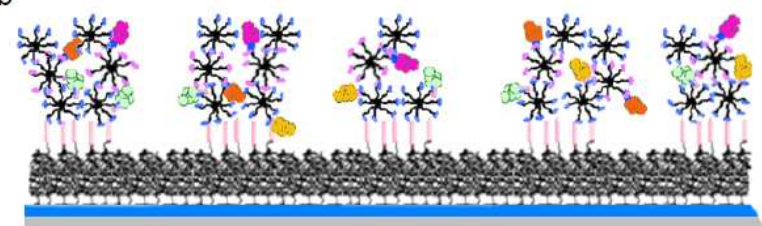

Figure 4. Illustration of the build-up of hydrogel-like structures. a) Suggested structure of the hydrogel-like stepwise immobilization measured by OWLS (Lys-PEG, TG-PEG and Lys-PEG). b) Conceptual image of a possible scenario with multifunctional patterned 3D hydrogel-like structures. 


\section{Conclusions}

We demonstrated an FXIIIa-catalyzed coupling scheme for site-specific tethering of ligands onto a surface that preserves the protein's bioactivity. Our surface modification method is based on the positioning of substrates for FXIIIa on a surface and their usage as initiator molecules for the further coupling of ligands. The ligands have been synthesized such that they comprised one of the counter reactive substrates for the enzyme. We showed that by using the previously described MAPL patterning technology, it is possible to have control over the spatial arrangement of the FXIIIa substrates on the surface. By applying a sitespecific FXIIIa-catalyzed enzymatic reaction it is also possible to translate this physical arrangement into a bioactive pattern, providing cells not only with adhesive-sites but also with morphogenes such as endothelial cell specific mitogen; TG-VEGF ${ }_{121}$. In addition, we provided evidence that this enzymatic approach allows a stepwise formation of more complex arrangements and even combinations of bioactive molecules. By recombinant protein expression and chemical synthesis a tool box with bioligands engineered to contain substrates for FXIIIa can be relatively easy established. These ligands when used together with our sitespecific surface immobilization technique will enable the formation of complex arrangements and combinations of bioactive molecules. We therefore believe that this is a versatile and powerful 2D platform to study cell-cell and cell-matrix interactions. 


\section{Acknowledgement}

This work was supported by the Competence Centre for Materials Science and Technology. 


\section{Supporting Information Available:}

PDF file containing four Figures. ${ }^{1} \mathrm{H}-\mathrm{NMR}$ analysis of the synthesized PLL- $g$-PEG-TG copolymer (Figure S1). In situ OWLS adsorption of PLL-g-PEG-TG on a titanium-coated waveguide and protein resistance of the adsorbed polymer layer $(0.3 \%$ BSA). The surface was also tested for incubation with calcium chloride $(25 \mathrm{mM})$ and its resistance to protein adsorption was again tested (3\% BSA) (Figure S2). A summary of the PLL-g-PEG-TG characterization by ${ }^{1} \mathrm{H}-\mathrm{NMR}$ and OWLS (molecular weight, grafting ratio, fraction of functionalized PEG, polymer adsorbed mass and its protein resistance); theoretical peptide densities based on experimental values (theoretical TG peptide surface densities, distance between the immobilized TG moieties, molecular weight of ligands used in the study and theoretical ligands surface densities) and theoretical immobilization rates on the surface (Figure S3). A representative in situ OWLS immobilization graphic; 8-arm Lys-PEG followed by TG-VEGF 121 (Figure S4). This material is available free of charge via the Internet at http://pubs.acs.org. 


\section{References}

1. Bottaro, D. P., Liebmann-Vinson, A., Heidaran, M.A. N.Y. Acad. Sci. 2002, 961, 143153.

2. Lutolf, M. P., Hubbell, J. A. Nat. Biotechnol. 2005, 23, 1, 47-55.

3. Hynes, R. O. Science. 2009, 326, 5957, 1216-1219.

4. Daley, W. P., Peters, S.B., Larsen, M. J. Cell Sci. 2007, 121, 3, 255-264.

5. Tibbitt, M. W., Anseth, K.S. Biotechnol. Bioeng. 2009, 103, 4, 655-663.

6. Griffith, L. G., Swartz, M. Nat. Rev. Mol. Cell Bio. 2006, 7, 211-224.

7. Rusmini, F., Zhong, Z., Feijen, J. Biomacromolecules. 2007, 8, 1775-1789.

8. Falconnet, D., Csucs, G., Grandin, H.M., Textor, M. Biomaterials. 2006, 27, 30443063.

9. Kane, R. S., Takayama, S., Ostuni, E., Ingber, D.E., Whitesides, G.M. Biomaterials. 1999, 20, 2363-2376.

10. Wolfram, T., Belz, F., Schoen, T., Spatz, J.P. Biointerphases. 2007, 2, 1, 44-48.

11. Kloxin, A. M., Kasko A.M., Salinas, C.N., Anseth, K.S. Science. 2009, 324, 59-63.

12. Lutolf, M. P. Integr. Biol. 2009, 1, 239-241.

13. Schuler, M., Hamilton, D.W., Kunzler, T.P., Sprecher, C.M., de Wild, M., Brunette, D.M., Textor, M., Tosatti, S.G. J. Biomed. Mater. Res., Part B. 2009, 91, 2, 517-527.

14. Massia, S. P., Hubbell, J.A. J. Cell Biol. 1991, 114, 1089-1100.

15. Cavalcanti-Adam, E. A., Aydin, D., Hirschfeld-Warneken, V.C., Spatz, J.P. HFSP J. 2008, 2, 5, 276-285.

16. DiMilla, P. A., Barbee, K., Lauffenburger, D.A. Biophys. J. 1991, 60, 15-37.

17. Palecek, S. P., Loftus, J.C., Ginsberg, M.H., Lauffenburger, D.A., Horwitz, A.F. Nature. 1997, 385, 537-540.

18. Brandley, B. K., Schnaar, R.L. Dev. Biol. 1989, 135, 74-86.

19. Maheshwari, G., Brown, G., Lauffenburger, D.A., Wells, A., Griffith, L.G. J. Cell Sci. 2000, 113, 1677-1686.

20. Irvine, D. J., Hue, K.A., Mayes, A.M., Griffith, L.G. Biophys. J. 2002, 82, 120-132.

21. Dee, K. C., Anderson, TT., Bizios., R. Tissue Eng. 1995, 1, 135-145.

22. Koo, L. Y., Irvine, D.J., Mayes, A.M., Lauffenburger, D.A., Griffith, L.G. J. Cell Sci. 2002, 115, 1423-1433.

23. Huang, J., Grter, S.V., Corbellini, F., Rinck, S., Bock, E., Kemkemer, R., Kessler, H., Ding, J., Spatz, J.P. Nano Lett. 2009, 9, 3, 1111-1116. 
24. Selhuber-Unkel, C., López-García, M., Kessler H., Spatz, J.P. Biophys. J. 2008, 92, 5424-5431.

25. Healy, K. E., Rezania, A., Stile, R.A. Ann. N.Y. Acad. Sci. 1999, 875, 24-35.

26. Maheshwari G., W., A., Griffith, L.G. Lauffenburger, D.A. Biophys. J. 1999, 76, 2814-2823.

27. Falconnet, D., Koenig, A., Assi, F., Textor, M. Adv. Funct. Mat. 2004, 14, 8, 749-756.

28. Tosatti , S., Schwartz, Z., Campbell, C., Cochran, D. L., VandeVondele, S., Hubbell, J.A., Denzer, A., Simpson, J., Wieland, M., Lohmann, C. H., Textor, M., Boyan, B. D. J. Biomed. Mater., Res. A. 2003, 68A, 3, 458-72.

29. Schuler, M., Owen, G.R., Hamilton, D.W., Wild, M., Textor, M., Brunette, D.M., Tosatti S.G.T. Biomaterials. 2006, 24, 4003-4015.

30. Gunawan, R. C., King, J.A., Lee, B.P., Messersmith, P.B., Miller, W.M. Langmuir. 2007, 23, 10635-10643.

31. Ichinose, J., Morimatsu, M., Yanagida, T., Sako, Y. Biomaterials. 2006, 27, 18, $3343-$ 3350 .

32. Fan, V. H., Au, A., Tamama, K., Littrell, R., Richardson, LL.B., Wright, J.W., Wells, A., Griffith, L.G. Stem Cells. 2007, 25, 1241-1251.

33. Mann, B. K., Schmedlen, R.H., West, J.L. Biomaterials. 2001, 22, 439-444.

34. Stefonek-Puccinelli, T. J., Masters, K.S. Ann. Biomed. Eng. 2008, 36, 12, 2121-2133.

35. Anderson, S. M., Chen, T.T., Iruela-Arispe, M.L., Segura, T. Biomaterials. 2009, 30, 4618-4628.

36. Hodneland, C. D., Lee, Y.S., Min, D.H., Mrksich, M. PNAS. 2002, 16, 5048-5052.

37. Sugimura, Y. U., H., Maki, M., Hitomi, K. J. Biotechnol. 2007, 131, 121-127.

38. Tominaga, J., Kamiya, N., Doi, S., Ichinose, H., Maruyama, T., Goto, M. Biomacromolecules. 2005, 6, 2299-2304.

39. Hu, B. H., Messersmith, P.B. J. Am. Chem. Soc. 2003, 125, 14298-14299.

40. Ichinose, A., Tamaki, T., Aoki, N. FEBS Lett. 1983, 153, 2, 369-371.

41. Ehrbar, M., Rizzi, S.C., Hluschchuk, R., Djonov, V., Zisch, A.H., Hubbell, J.A., Weber, F.E., Lutolf, M.P. Biomaterials. 2007, 28, 26, 3856-3866.

42. Ehrbar, M., Rizzi, S.C., Shoenmakers, R.G., San Miguel, B., Hubbell, J.A., Weber, F.E., Lutolf, M.P. Biomacromolecules. 2007, 8, 10, 3000-3007.

43. Lorand, L., Conrad, S.M. Mol. Cell. Biol. 1984, 58, 9-34.

44. Cleary, D. B., Maurer, M.C. Biochim. Biophys. Acta 2006, 1207-1217.

45. Lorand, L., Graham, R.M. Nat. ReV. Mol. Cell Biol. 2003, 4, 2, 140-156. 
46. Pasche, S., De Paul, S.M., Vörös, J., Spencer, N.D., Textor, M. Langmuir. 2003, 19, 22, 9216-9225.

47. VandeVondele, S., Vörös, J., Hubbell, J.A. Biotechnol. Bioeng. 2003, 82, 7, 784-790.

48. Tosatti, S., De Paul, S.M., Askendal, A., VandeVondele, S., Hubbell, J.A., Tengvall, P., Textor, M. Biomaterials. 2003, 24, 4949-4958.

49. Lutolf, M. P., Hubbell, J.A. Biomacromolecules. 2003, 4, 3, 713-722.

50. Vörös, J., Ramsden, J.J., Csucs, G., Szendro, I., De Paul, S.M., Textor, M., Spencer, N.D. Biomaterials. 2002, 23, 17, 3699-3710.

51. Lussi, J. W., Falconetti, D., Hubbell, J.A., Textor, M., Csucs, G. Biomaterials. 2006, 27, 2534-2541

52. Christinger H.W., M., Y.A., Berleau, L.T., Keyt, B.A., Cunningham, B.C., Ferrara, N., de Vos, A.M. PROTEINS. 1996, 26, 353-357.

53. Muller, Y. A., Li, B., Christinger, H.W., Wells, J.A., Cunningham, B.C, de Vos, A.M. Proc. Natl. Acad. Sci. USA. 1997, 94, 7192-7197. 
Table of contents only

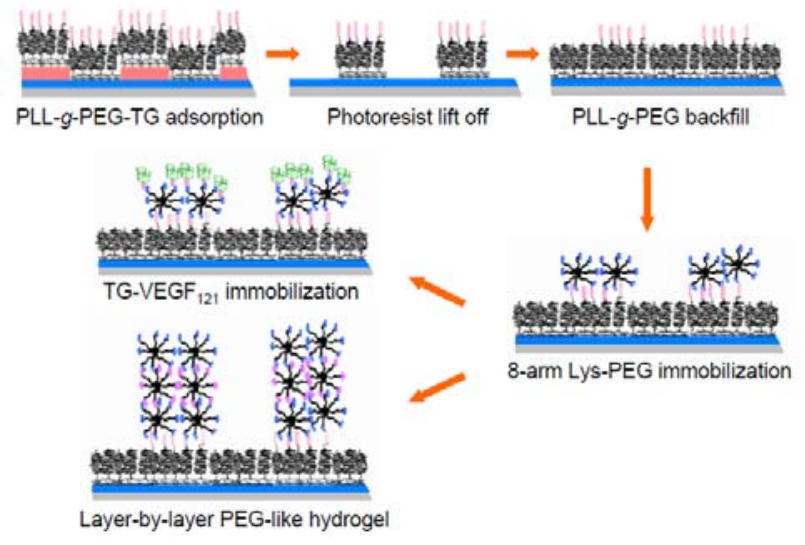

